\title{
Tolerance of Training and Competitive Loads in Sports on the Basis of Psychological Type: Theoretical Literature Review
}

\author{
Zurin E.A. \\ Federal Science Center of Physical Culture and Sport \\ Moscow, Russia
}

\author{
Shuvalova L.S. \\ Federal Science Center of Physical Culture and Sport \\ Moscow, Russia
}

\author{
Kryuchkov A.S. \\ Federal Science Center of Physical Culture and Sport \\ Moscow, Russia
}

\begin{abstract}
The author of this scientific research is greatly interested in ski racing and the problem of rational loads distribution by volume and intensity during the annual training cycle. This article discusses relationship between the tolerance of training and competitive loads in sports with the psychotype of an athlete. The analysis of literature showed that this problem is insufficiently explored. We have identified areas of brain cortex, decreased activity of which, in case of irrationally chosen training and competitive loads, affects the subjective severity of load perception by an athlete as well as the effectiveness of realization.
\end{abstract}

Keywords - Load tolerance; training and competitive loads; limit loads; individualization of the training process; adaptation; recovery; psychotype; type of temperament; behavior; motor activity; 'Human Genome'; neurotransmitters; neuromodulators; neuroticism; extroversion; regulatory zones of brain cortex; decreased $\mathrm{pH}$ of muscles and blood.

\section{INTRODUCTION}

In modern high-level sport, when the requirements imposed on the systems of athlete's body become closer to the individual limit of loads tolerance, search for new methodological approaches and directions to improve the efficiency of athletes' performance in favorite sports continues to be relevant. One of such directions is individualization of the training process on the basis of adaptation peculiarities of an athlete's body to the offered training and competitive loads depending on their individual psychotype.

\section{LITERATURE REVIEW}

The problem of training tolerability and competitive loads in sports is considered from the point of view of balance between the limit exertion of functional capabilities of the athlete's body and the optimal recovery period (V.A. Ponomareva, L.V. Safonova, 2015 etc.), as well as the use of rehabilitation measures that allow to form a new level of the adaptation functional system to physical loads (V.A. Pankov, 2000; V.N. Platov, 2004; A.E. Donnikova, 2009; A.S. Kulemina, 2013; R.V. Khomenko, 2017 etc.). Research of tolerability of training and competitive loads in sports on the basis of psychotypes of athletes' features is presented in the research of Drizhik Anatoly Georgievich in the context of qualified athletes' preparation process individualization. The peculiarities of athletes' psychotypes during composition of preparatory training microcycles were considered by B.N. Shustin. For introverted athletes, characterized by inertia, predominance of inhibitory processes, effective can be microcycles, which use intensive exercises with an emotional nature of work. And for athletes of the extroverted personality type, with prevailing processes of excitation, microcycles with a soft mode of work, a small volume of intensive exercises, rather low loads during separate trainings are apropriate [8]. In scientific and methodical literature, characteristics of individual features of psychotypes are presented from the point of view of researching the forms of behavior peculiar to them, ways of information perception and decision-making. These characteristics are determined by hormonal profile, balance between the actions of stimulating (stimulating the brain cortex) and calming (inhibiting) neurotransmitters, by strength and weakness of nervous processes, their mobility and equability, dynamism and lability, as well as by general and partial properties of the nervous system [6,11]. It is necessary to take into account to what extent is expressed the need for communication and interaction with other people (extroversion-introversion), neuroticism - emotional susceptibility and irritability, tendency to mood changes, personal and situational anxiety. The degree of expressiveness and combination of these indicators forms the basis of the modern approach to classification of human temperament types - choleric, sanguine, phlegmatic, melancholic $[2,6,7,10]$. On the basis of research data within the framework of the international program "Human Genome", the characteristics of psychotypes indicated by us are genetically predetermined and the indicators of heritability of these character traits are 30$60 \%$. It should be noted that the individual characteristic of a person within the 4 types of temperaments is conditional. In actual fact, we should speak about the leading type of temperament in the structure of personality $[1,2,7,11]$. The 
nature of this phenomenon is expressed by a close relationship of the psychotype components with different areas of the brain cortex involved in the regulation of motor, cognitive and emotional processes. The regulatory zones of brain cortex include parts of the prefrontal cortex (dorsolateral prefrontal cortex, orbitofrontal cortex, posterior parietal cortex), basal nuclei and limbic system of the brain [6, 12]. Due to the executive functions of the orbitofrontal cortex, an athlete has the ability to plan current actions in accordance with the general goal, change the reaction depending on the situation, selectively pay attention to the necessary stimuli, ensuring cognitive flexibility (for example, use a variety of tactical maneuvers when covering segments of the distance) [12]. The orbitofrontal cortex is the key structure responsible for adaptive training [14], and it provides an assessment of the subjective emotional experience [12].

Sustaining the optimal activity level of the prefrontal cortex allows an athlete to maintain the capability to perform motor actions under the specified parameters in the context of various interferences, i.e. to exercise volitional control. This capability is particularly important for highly qualified athletes during trainings in conditions of a significant $\mathrm{Ph}$ decrease of muscles and blood, with the utmost intensity (at the level of maximal oxygen consumption). This state of body occurs as a result of high intensity of muscular work requiring mobilization of the psyche. As a result, fatigue develops in the cortex of the large hemispheres, which is expressed in the inability to maintain sufficient intensity of trainings (thus, volitional control reduces), resulting in a significant loss of productivity. This state of CNS fatigue is quite critical for an athlete, because with the reduced productivity, being inactive for a week, one can lose up to $10 \%$ of aerobic capacity, and a little less percentage of strength [5].

In addition to the prefrontal area of brain cortex, a special role is played by brain structures included in the limbic system. These structures determine the choice and realization, as well as the dynamics of inborn and adaptive forms of human behavior, ensure the maintenance of homeostasis [6]. Due to its effect on the hypothalamus, and through it on the hormonal profile of the body (on the components of serotoninergic, dopaminergic, noradrenaline and reninangiotensive hormonal systems), the limbic system participates in the formation of impulses to action (motivations) and its implementation, strengthening or weakening emotional reactions and behavior factors $[1,7,13]$. In the structure of the limbic system, in addition to its other components, the anterior cingulate cortex (ACC) should be distinguished. Cognitive functions of the anterior cingulate cortex are associated with the prefrontal cortex and parietal cortex, as well as the motor system and frontal ophthalmic fields, making it a central site for processing "descending" (associated with goal-setting and motivation) and "ascending" (associated with momentary decisions) stimuli and sending control signals to other areas of the brain. The anterior cingulate cortex determines the emotional component in human behavior, being in close relationship with other brain structures - the amygdala, nucleus accumbens, hypothalamus and anterior insular cortex. In interaction with the prefrontal cortex of the large hemispheres, it provides the ability of the psyche to respond emotionally to various stimuli, provides selectivity and concentration of attention, participates in the formation of motivation for activity [14]. Changes in the level of activity of the anterior cingulate cortex towards a decrease in its indicators leads to changes in the motivational and volitional components of the psyche. Fatigue of the limbic system areas leads to a deficit of attention and to a decrease in coordination abilities, the quality of performing technical elements in the structure of motor activity. In case of anterior cingulate cortex fatigue, the function of emotional assessment of external stimuli impact on the psyche also undergoes distorting, the usual volume and intensity of physical activity may be interpreted as submaximal or critical, the level of achievement motivation reduces and the level of failure avoiding motivation may increase $[6,10]$.

Components of the limbic system, basal nuclei and prefrontal cortex zones are interconnected; changes in the activity of these structures have its influence on the functioning of other parts of the brain, including the cerebellum. It is exactly the cerebellum activity that is associated with the regulation of posture and muscle tone, sensomotor coordination of postural and purposive movements, coordination of fine movements. As a result of fatigue and decreased activity of the brain areas associated with the work of the cerebellum, we can observe change in the indicators of its effectiveness as a coordinator of motor acts which integrates and gives commands for the implementation of both simple and complex voluntary movements; the technique of building a movement changes. In case of excessive fatigue of brain areas that affect the activity of the cerebellum, its functionality as a coordinator of various motor actions reduces. The indicators of rationality and efficiency of sports technique, the level of sports and technical skill of athletes change. The risk of injury of the musculo-skeletal system of an athlete increases.

\section{RESEARCH METHODOLOGY}

The demonstration of any physical quality, whether that be strength, speed, flexibility, agility or endurance, is unthinkable without the display of mental component, which in combination with movement forms the psychomotor structure of activity, based on the unity of target, semantic and motor components [3]. Accordingly, it is incorrect to study and analyze the psychological aspect of an individual in isolation from the functional state of the organism. Herewith it should be noted that the main purpose of sports activities of athletes is to achieve maximum sports results. Sports result in this context can be considered with regard to the product of movements and translocations of an athlete, reflection of efficiency of motor potential in specific conditions of activity [9]. The sportsman can consciously change his behavior in response to interferences, for example, when there is a significant decrease in the $\mathrm{Ph}$ of his muscles and blood, but he does not stop running to the intended target and achieves the result. Here we can see involvement not only of functional systems, but also motivational, volitional components of the personality. It becomes obvious that the psychological component cannot be removed either from the motor activity or from the sports result. 


\section{RESULTS}

As a result of the scientific-methodical literature theoretical analysis on the problem of tolerance to training and competitive loads we have identified evidence speaking for individual peculiarities of athletes' psyche which play an important role in the process of body adaptation and recovery after training and competition loads in sport.

Genetic conditioning of characteristics of the nervous system properties (including sympathetic and parasympathetic), of stimulating and inhibiting neurotransmitters (glutamate, acetylcholine, gammaaminobutyric acid, glycine) and neuromodulators (norepinephrine, serotonin, adenosine, dopamine), of higher nervous activity types suggests the most optimal types of psychological constitution for some sports. Such sport as skiing imposes a number of requirements on athlete's psyche, the main of which is the ability to tolerate extreme and nearextreme mental and physical loads [5]. According to the opinion of I.I. Akhmetov, in cyclic kinds of sports, especially concerning long distances, the most successful will be an athlete possessing a psychotype with a leading type of temperament "phlegmatic". This psychological type of athlete constitution is characterized by emotional stability, average level of activity and weak switch of attention, it shows a high level of efficiency under the conditions of monotonous activity [1].

At short and medium distances, the most effective will be the psychotype with the leading type of temperament "sanguine". At short distances - "choleric" type. Emotional stability, high speed of response, typical of sanguine type, allow to cope with large and intense amounts of physical exercises, but on condition that their activity will include a variety of episodes, primarily aimed at attention switching. Emotional reactions of choleric type, on the contrary, are less stable due to the dominance of excitation processes in the subcortical structures of the brain. This psychotype has a quick reaction rate and is able to tolerate extreme or nearextreme loads on the premise of increased achievement motivation and with obligatory "bright" speedy inserts.

\section{DISCUSSION}

Adaptation features of the organism of athletes with different psychotypes to training loads can be revealed as a result of: cycle;

- dynamic observations throughout the annual training

- research of distribution features of various loads by volume and intensity;

- determination of biochemical characteristics;

- analysis of indicators of functional state and subjective perception of the severity of the training load by an athlete.
Herewith it is necessary to analyze in detail the types, structure of used microcycles, applied method of building the training process and specific tools in training and competitive activities.

\section{CONCLUSION}

We believe that as a promising direction in programming of the training process in accordance with generally accepted principles of planning and management (registration of functional state of organism, genetic features, biochemical processes, individual characteristics etc.) it is necessary to take into account the psychological type of an athlete constitution, leading traits of personality in relation to tolerance of training loads of different orientation in annual cycle of preparation and the characteristics of the flow of adaptation processes.

\section{References}

[1] Akhmetov, I.I. Molecular genetics: monograph. Moscow: Soviet sport. 2009, 268

[2] Eysenck G. Personality Structure. SPb.: Juventus; M.: KSP+. 1999, 464.

[3] Verkhoshansky, Y.V. Fundamentals of special physical preparation of athletes. Moscow: physical Culture and sport. 1988, 331.

[4] Lemeshko V.S. Athletic performance as a multifactorial phenomenon in the training of highly qualified Skorokhodov. Vestnik Mazurskie zargari pedagogichnogo norsat IMIA I.P. Shamyakina. 2012.

[5] Manzhosov V.N. Principles of preparation of the skier-racer. Theory and practice of physical culture. 1992, 1, pp. 2-4.

[6] Lebedev A.A., Rusanovskiy V.V., Lebedev V.A., Shabanov P.D. Neurophysiology. Fundamentals of the course: textbook. Moscow: KNORUS. 2019, 232.

[7] Rusalov V.M, Trofimova I.N. About the representation of types of mental activity in different models of temperament. Psychological journal. 2011, vol. 32/3, pp. 74-84.

[8] Suslov F.P., Sych V.L., Shustin B.N. Modern system of sports training. Moscow: SAAM. 1995, 445.

[9] Solodkov A.S., Sologub E.B. Human physiology, General, Sports, Age. 2005.

[10] Brain SPECT Imaging in Complex Psychiatric Cases: An EvidenceBased, Underutilized Tool, Amen D.G., Hanks C., Prunella J. Predicting positive and negative treatment responses to stimulants with brain SPECT imaging. Psychoactive Drugs. 2008, 40, pp.131 - 8 .

[11] Cloninger C.R., Svrakic D.M., Przybeck TR. A psychobiological model of temperament and character. Arch Gen Psychiatry. 1993, 50, pp. 975 $-990$.

[12] Rolls E.T., Grabenhorst F. The orbitofrontal cortex and beyond: from affect to decision-making. J Progress in Neurobiology. 2008, vol. 86, 3 , pp. 216-244. DOI:10.1016/j.pneurobio.2008.09.001.

[13] Trofimova I.N., Robbins T.W. Temperament and arousal systems: a new synthesis of differential psychology and functional neurochemistry. 2016, vol. 64, pp. 382-402. DOI:10.1016/ j.neubiorev.2016.03.008.

[14] Alesandr S. Kuznetsov, Evgeniya N. Usmanova, Oksana V. Kolomytseva. Athletes' psychological-physiological indices study in different specializations at the stages of sports carreer crises overcoming. Russian Journal of Physical Education and Sport. 2019, 14(2), pp. 75-81. DOI: 10.14526/2070-4798-2019-14-2-89-96

[15] Volz K.G. Cortical areas activated by the subjective sense of perceptual coherence of environmental sounds: A proposal for a neuroscience of intuition: Cognitive, Affective, \& Behavioral Neuroscience. 2008, vol. 8, 3, pp. 318-328. DOI:10.3758/CABN.8.3.318.]. 